\title{
Kleine Ringe erweitern: Auf die Spannung kommt es an
}

\author{
Johannes Kaschel, Daniel B. Werz
}

Wenn es gilt, komplexe Strukturen aufzubauen, erfreuen sich Kleinringsysteme schon seit langem großer Beliebtheit. Die Thermodynamik steht dabei auf der Seite des Synthetikers. Entscheidend für den Erfolg solcher Reaktionen ist nur das richtige Werkzeug, um den Ring zu öffnen.

Die kleinsten und damit gespanntesten Kohlenstoffzyklen sind das Cyclopropan und das Cyclobutan. Mit ihren Gesamtringspannungen von $115 \mathrm{bzw} .101 \mathrm{~kJ} \cdot \mathrm{mol}^{-1}$ sind sie für Ringöffnungs- und Ringerweiterungsreaktionen prädestiniert. Dass Cyclopropane trotz ihrer hohen Ringspannung doch einigermaßen stabil sind, erklärt am besten das Walsh-Modell. ${ }^{1)}$ Es geht von $\mathrm{sp}^{2}$-hybridisierten Kohlenstoffatomen aus - untermauert durch die ${ }^{1} J_{\mathrm{CH}}$-Kopplungskonstanten von etwa $160 \mathrm{~Hz}$ und die Ähnlichkeit zur Reaktivität von Olefinen. Dennoch sind Cyclopropane - insbesondere wenn sie Reste wie Donor-Akzeptor-Substituenten tragen oder Übergangsmetalle ihre Reaktion katalysieren - sehr reaktiv und neigen zu spannungsabbauenden Reaktionen. Die auf diese Weise schnell zugänglichen, größeren Carbo- oder Heterozyklen machen Cyclopropane zu nützlichen Substraten in der Synthese. ${ }^{2)}$ Auch das höhere Homologe, das Cyclobutan, geht leicht Ringerweiterungsreaktionen ein. In jüngerer Zeit haben auch Vierringsysteme, deren Ringspannung für Folgereaktionen benutzt wird, an Bedeutung gewonnen.

\section{Donor-Akzeptor-Substitution}

- Ein elektronenschiebender (Donor, D) und ein elektronenziehender

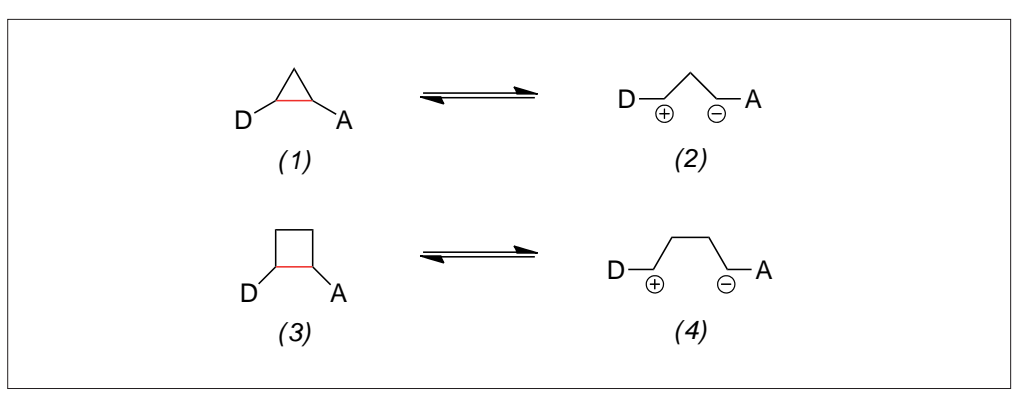

Abb. 1.

Grenzstrukturen Donor-Akzeptorsubstituierter Cyclopropane und -butane. (rot: Bindung, die geschwächt wird)

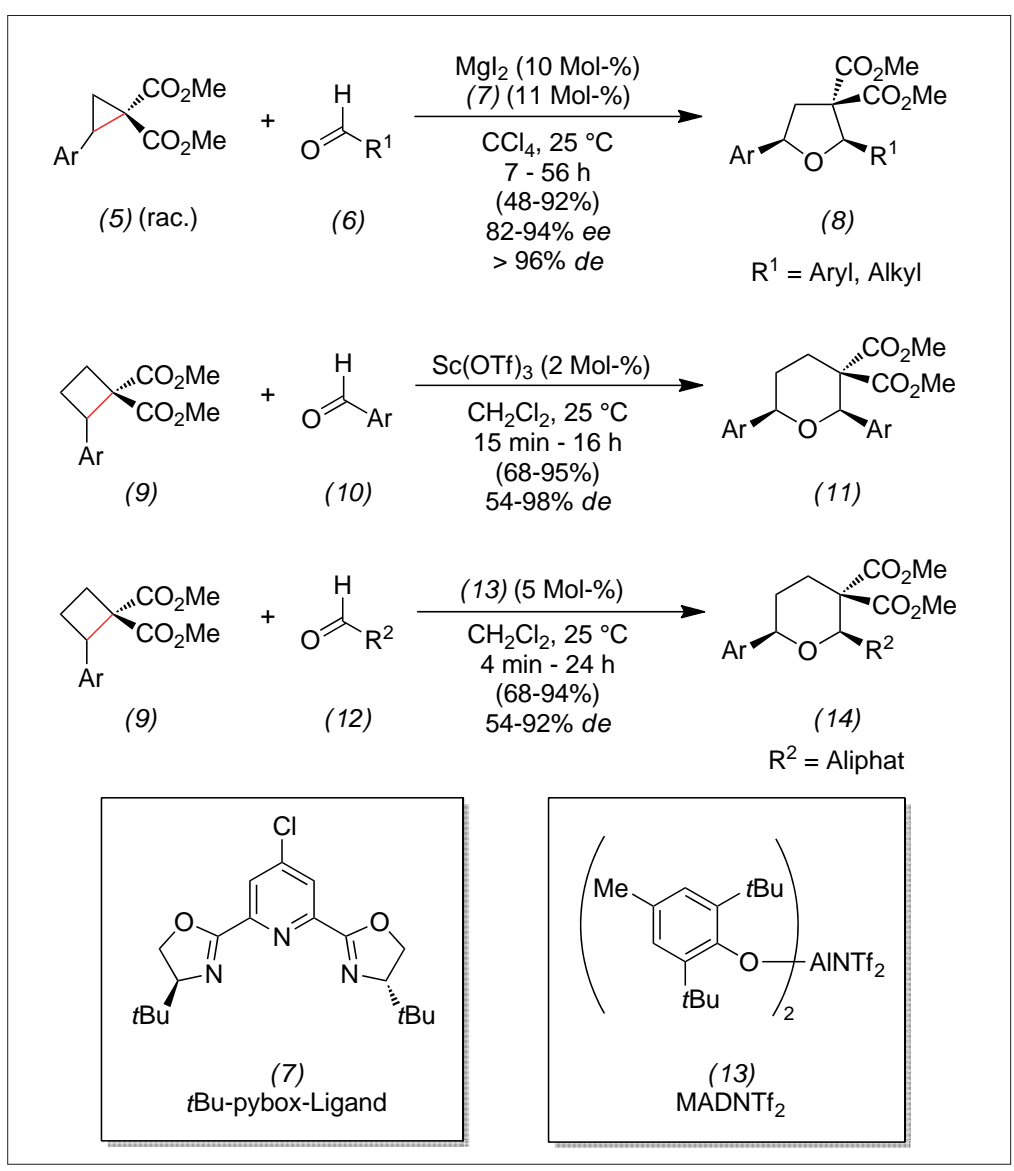

Abb. 2.

Synthese von Tetrahydrofuranund Tetrahydropyran-Derivaten nach Johnson et al. 
(Akzeptor, A) Substituent in vicinaler Position schwächen die zentrale, in Abbildung 1 rot gekennzeichnete Bindung des Cyclopropans (1) und -butans (3) so stark, dass sich die 1,3- bzw. 1,4-dipolaren Grenzstrukturen (2) bzw. (4) formulieren las- sen. Die Zugabe von Lewis-Säuren, die an den Akzeptor-Substituenten koordinieren, schwächt die Bindung noch weiter.

Die Verwendung der dipolaren Spezies (2) und (4) für [3+2]- bzw. [4+2]-Cycloadditionen ist eine lange

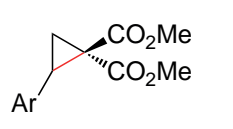

(5)<smiles>[R]c1ccc2cc(C#C)n([R])c2c1</smiles>

(15)

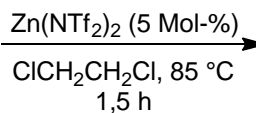 $(49-90 \%)$}

$\mathrm{R}^{1}=\mathrm{CF}_{3}, \mathrm{CO}_{2} \mathrm{Me}, \mathrm{Me}$ $\mathrm{R}^{2}=\mathrm{H}, \mathrm{Me}, \mathrm{Bn}$

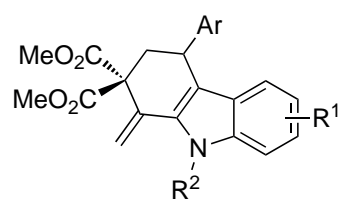

(16)

Abb. 3. Ringöffnungsreaktion des Cyclopropans gefolgt von einer Conia-En-Zyklisierung zum Aufbau von Tetrahydrocarbazolen nach Kerr et al.

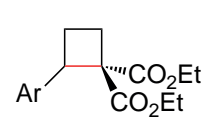

(17)<smiles>C1=COCCC1</smiles>

(20)

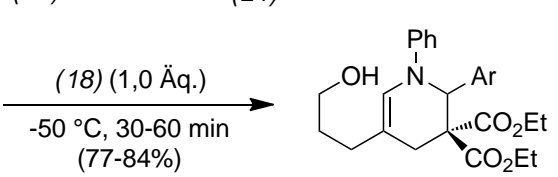

(23)

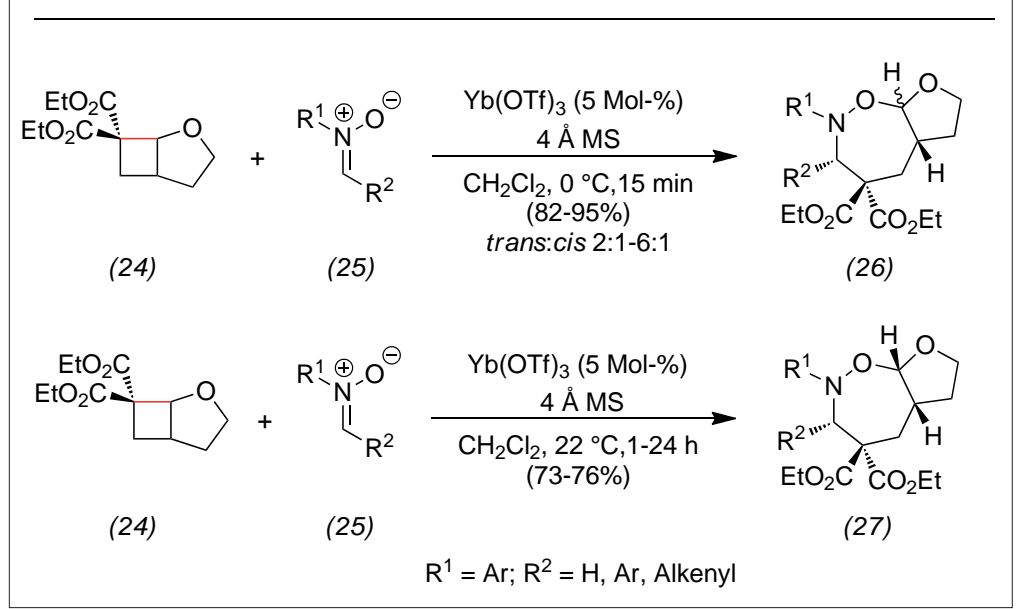

Abb.4. Cycloadditionen von Donor-Akzeptor-Cyclobutanen mit Iminen und Nitronen nach Pagenkopf et al. bekannte Möglichkeit, um Fünfund Sechsringe aufzubauen. Heutzutage stehen bei Reaktionen dieser Art meist Fragen der Stereoselektivität im Vordergrund. So entwickelten Johnson et al. eine effiziente Methode, um aus racemischen Cyclopropanen (5) in einer asymmetrischen [3+2]-Cycloaddition mit Aldehyden (6) stark enantiomerenangereicherte Tetrahydrofuran-Derivate (8) herzustellen. Ein Katalysator für diese dynamisch-kinetische Racematspaltung sollte einerseits selektiv die Cycloaddition des Aldehyds mit nur einem Enantiomer des Cyclopropans und andererseits die Umwandlung der Enantiomere vermitteln. Dazu kam Magnesiumiodid in Kombination mit einem tert-Butyl-pybox-Liganden (7) zum Einsatz. J) Johnson zeigte außerdem, dass auch Cyclobutane vom Typ (9) in einer ähnlichen Reaktion zu den entsprechenden Tetrahydropyran-Derivaten (11) bzw. (14) umgesetzt werden können. Besonderes Augenmerk lag hier auf der Verwendung von kohlenstoffbasierten Donoren am Cyclobutan; ursprünglich wurden heteroatomare Gruppen als bessere Donoren eingesetzt. Als besonders geeignet erwies sich für diese Reaktion Scandium(III)triflat als katalytisch wirkende Lewis-Säure. Die Ausbeuten betrugen $68-96 \%$ bei einem Diastereomerenüberschuss von 54-98\%. Für die Umsetzung aliphatischer Aldehyde (12) musste MADNTf $_{2}$ (13) als Katalysator verwendet werden (Abbildung 2). ${ }^{4)}$

Auch sechsgliedrige Zyklen lassen sich durch die Anlagerung von Donor-Akzeptor-substituierten Cyclopropanen (5) gewinnen. Ausgehend von 2-Alkinylindolen (15) nutzten Kerr et al. eine nucleophile Ringöffnungsreaktion, gefolgt von einer Conia-En-Cyclisierung, um Tetrahydrocarbazole (16) aufzubauen. $\mathrm{Zn}\left(\mathrm{NTf}_{2}\right)_{2}$ erwies sich als am besten geeignete Lewis-Säure, um sowohl das Cyclopropan für die Ringöffnung als auch das Alkin für den Conia-En-Prozess zu aktivieren (Abbildung 3). ${ }^{5)}$

Über weitere Beispiele für die Ringöffnung von D-A-Cyclobutanen 
berichteten in letzter Zeit Pagenkopf et al. Eine formale [4+2]-Cycloaddition mit in situ gebildeten Iminen (18) lieferte unter Ytterbium(III)triflat-Katalyse Piperidine (19) und Tetrahydropyridine (23). Bei den Piperidinen wurde eine hohe trans-Selektivität der beiden dem Stickstoff benachbarten Gruppen beobachtet. Da sich der gleiche Katalysator auch zur Herstellung alkoxysubstituierter Cyclobutane (22) eignet, war die Synthese von Tetrahydropyridinen (23) in einem Eintopfverfahren ausgehend von einem Enolether wie (20) und Methylen-Malonat (21) durchführbar. ${ }^{6)}$ Ebenfalls katalysiert durch $\mathrm{Yb}(\mathrm{OTf})_{3}$ führte Pagenkopf [4+3]-Cycloadditionen zwischen D-A-Cyclobutanen (24) und Nitronen (25) durch. Auf diese Weise sind Oxazepine (26) in Ausbeuten von bis zu $95 \%$ zugänglich. Die Veränderung von Temperatur und Reaktionszeit steuert die Stereochemie des Produkts. Bei tiefen Temperaturen und einer kurzen Reaktionszeit $\left(0^{\circ} \mathrm{C}, 15 \mathrm{~min}\right)$ entstand sowohl das trans- als auch das cis-Produkt, während sich bei Raumtemperatur und einer längeren Reaktionszeit ausschließlich das thermodynamisch günstigere cis-Produkt (27) bildete (Abbildung 4). ${ }^{7)}$

\section{Metallinsertionen}

Auch die leicht erfolgenden Insertionen von Metallen in die C-C-Bindung von Cyclopropanen und Cyclobutanen sind eine Folge der hohen Ringspannung. Die so erhältlichen, meist hoch reaktiven Metallacyclobutane sind ideale Intermediate für Folgereaktionen. Im Jahr 2007 führte Mascareñas neuartige palladiumkatalysierte intramolekulare [4+3]-Cycloadditionen von Alkylidencyclopropanen mit Dienen (28) durch. Mechanistisch ging er dabei zunächst von der Bildung des Palladacyclohexan-Intermediats (30) aus, das durch eine allylische Umlagerung das Palladacyclooctan-Intermediat (31) liefert. Letzteres ergibt nach reduktiver Eliminierung des Palladiums das aus einem Fünf- und einem Siebenring

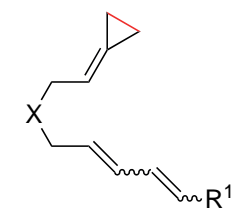

(28)

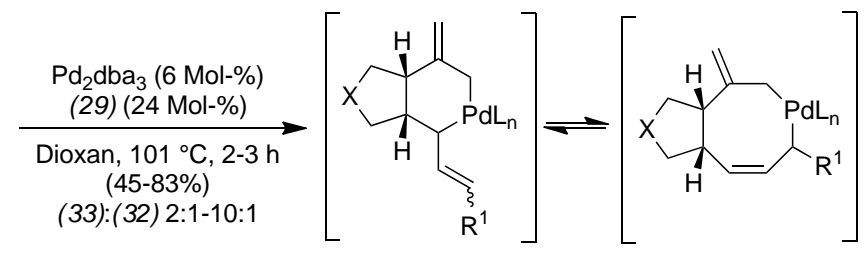

(30)

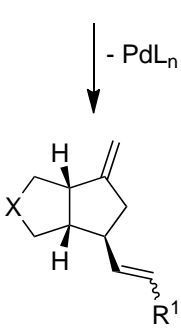

(32)

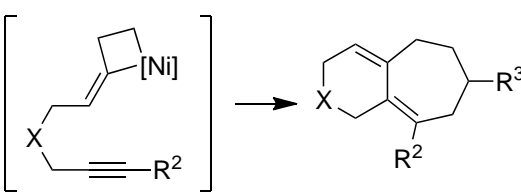

(36)
(31)
$X=\mathrm{O}, \mathrm{NBn}, \mathrm{C}\left(\mathrm{CO}_{2} \mathrm{Et}\right)_{2}$ $\mathrm{R}^{1}=\mathrm{H}, \mathrm{Ph}, \mathrm{CO}_{2} \mathrm{Et}$
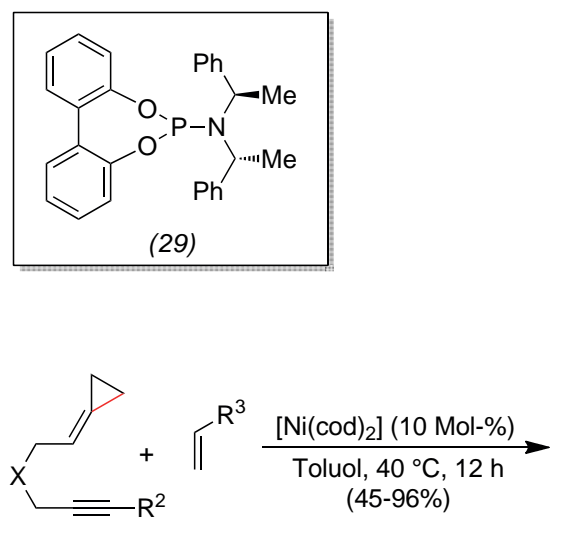

(34)
(35)

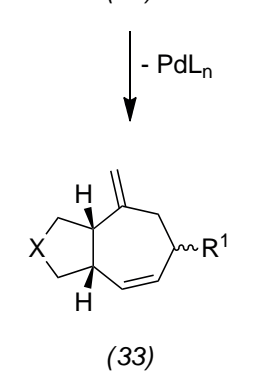

(33)
$\mathrm{X}=\mathrm{O}, \mathrm{NTs}, \mathrm{C}\left(\mathrm{CO}_{2} \mathrm{Et}\right)_{2}$

$\mathrm{R}^{2}=\mathrm{CO}_{2} \mathrm{Et}, \mathrm{CH}_{2} \mathrm{OAc}, \mathrm{CH}_{2} \mathrm{OTBS}, \mathrm{Me} ; \mathrm{R}^{3}=\mathrm{COMe}, \mathrm{CO}_{2} \mathrm{Et}, \mathrm{COH}, \mathrm{SO}_{2} \mathrm{Ph}$

(37)

Abb. 5. Palladium- und nickelkatalysierte Cyclopropan-Erweiterungsreaktionen nach Mascareñas et al.

bestehende bicyclische Produkt (33). Die Menge des entstehenden Nebenprodukts (32), das durch reduktive Eliminierung aus dem Palladacyclohexan-Intermediat (30) entsteht, ließ sich mit PhosphoramiditLiganden wie (29) verringern. ${ }^{8)} \mathrm{Ni}$ ckelkatalysiert gelang es Mascareñas außerdem, Alkylidencyclopropane (34) einer formalen [3+2+2]-Cycloaddition mit terminalen Alkenen (35) zu unterwerfen. ${ }^{9)}$ Dichtefunktionaltheorie-Rechnungen legen nahe, dass hierfür zunächst als Intermediat ein 1-Alkyliden-2-Nickelacyclobutan (36) gebildet wird, bevor das bicyclische System (37) entsteht (Abbildung 5).

Eine enantioselektive Umsetzung von Allenylcyclobutanolen (38) zu hochsubstituierten Cyclohexenonen mit quartären Stereozentren führten Cramer et al. in Gegenwart eines Rhodium(I)-Katalysators durch. Cramer nahm an, dass das Substrat

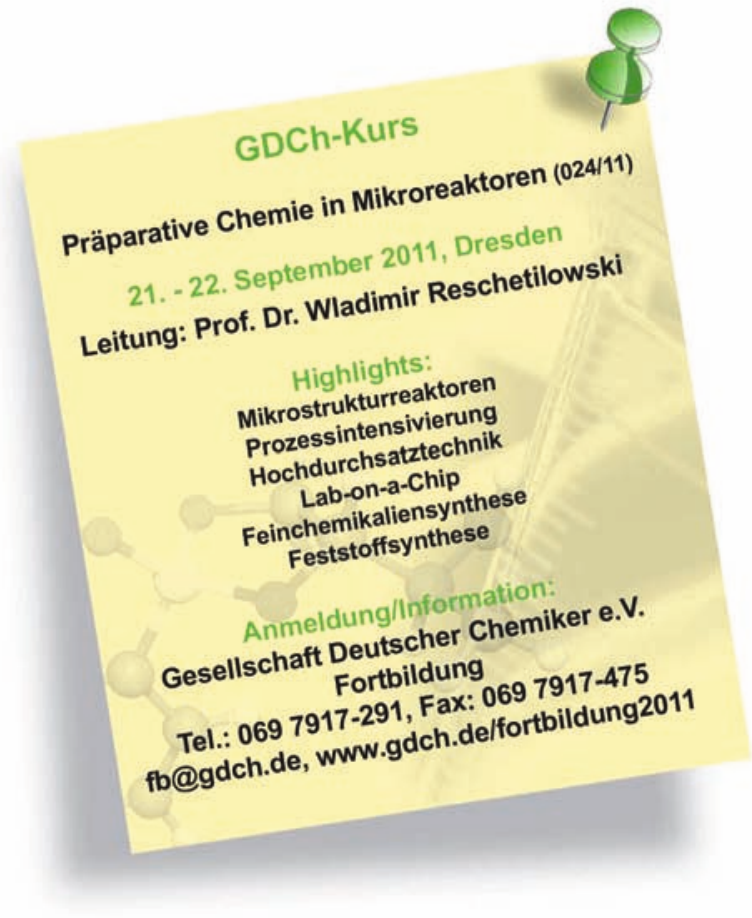




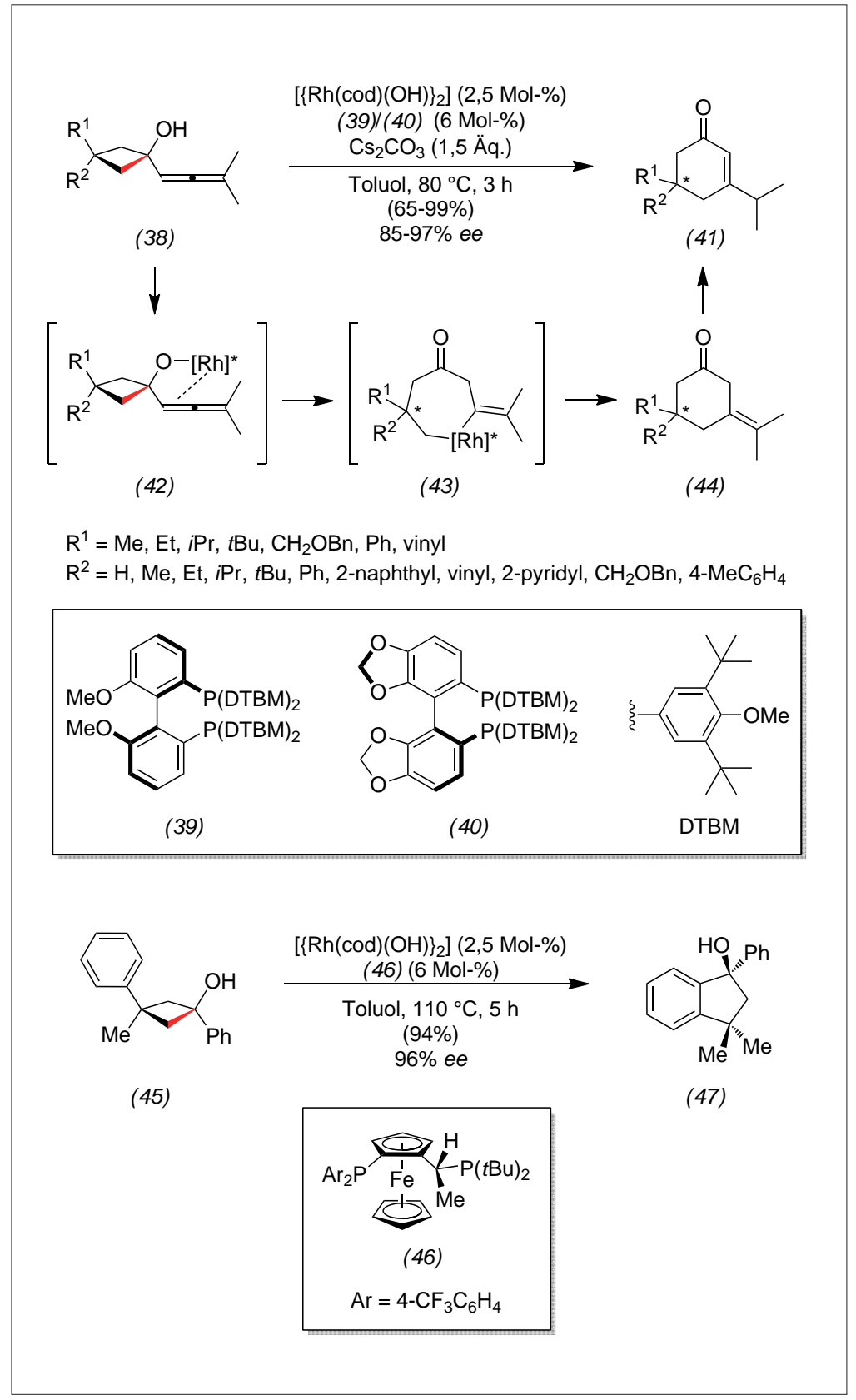

Abb. 6. Enantioselektive C-C-Bindungsaktivierung von Allenylcyclobutanolen (oben) und verwandte Indanol-Synthese (unten) nach Cramer et al.

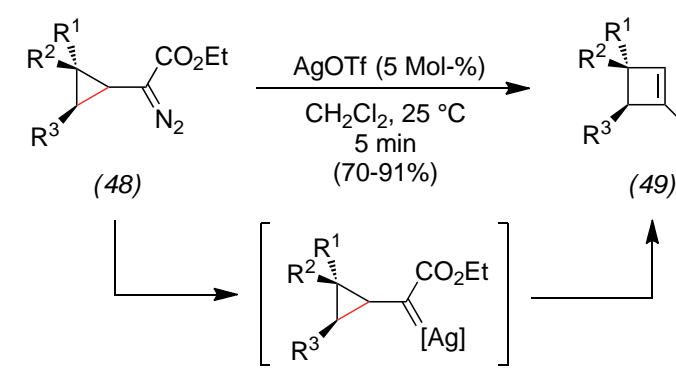

(50)

$\mathrm{R}^{1}=\mathrm{H}, \mathrm{CO}_{2} \mathrm{Et} ; \mathrm{R}^{2}=\mathrm{Pr}, \mathrm{CO}_{2} \mathrm{Me}, \mathrm{CO}_{2} \mathrm{Et}$ $\mathrm{R}^{3}=\mathrm{H}, \mathrm{Me} ; \mathrm{R}^{2}-\mathrm{R}^{3}=\mathrm{C}_{4} \mathrm{H}_{8}, \mathrm{C}_{6} \mathrm{H}_{12}, \mathrm{C}_{4} \mathrm{H}_{6}$

Abb. 7. Umlagerung von Cyclopropyl-Metall-Carbenen zu Cyclobuten-Derivaten nach Tang et al.
(38) wegen seines hohen Chelatisierungspotenzials, ausgehend von der Hydroxy- und der Allenfunktionalität, mit dem chiralen Rhodium(I)Katalysator ein Addukt (42) bildet und auf diese Weise die chirale Information übertragen wird. Die enantioselektive Insertion des Metalls in eine C-C- $\sigma$-Bindung des Cyclobutans führt dann zu einem siebengliedrigen Metallazyklus (43), der nach reduktiver Eliminierung das Methylen-Cyclohexanon (44) liefert. Das Zielprodukt (41) bildet sich im Anschluss durch Isomerisierung der semicyclischen Doppelbindung (Abbildung 6, oben). ${ }^{10)}$

Eine ähnliche Reaktion konnte zum stereoselektiven Aufbau von Indanolen (47) mit quartären Stereozentren genutzt werden. Sie beginnt ebenfalls durch die Insertion des Rhodiums in den Cyclobutanring, kommt dann aber durch eine C-H-Aktivierung am benachbarten Phenylring zum Abschluss (Abbildung 6 , unten). ${ }^{11)}$

\section{Benachbarte Carbene und Carbenoide}

- Eine weitere Möglichkeit, Umlagerungen oder Ringerweiterungen an Cyclopropanen einzuleiten, besteht darin, Carbene oder Carbenoide in direkter Nachbarschaft zum Kleinringsystem zu generieren. Ausgehend von leicht zugänglichen Cyclopropyldiazo-Verbindungen (48) nutzten Tang et al. die Abspaltung von Distickstoff in Gegenwart eines Silber-Katalysators, um Cyclopropyl-Metall-Carben-Komplexe (50) zu erzeugen. Die 1,2-Wanderung einer C-C- $\sigma$-Bindung des Cyclopropans zum elektronenarmen Carbenkohlenstoff ergab dann selektiv das hoch substituierte Cyclobuten (49) (Abbildung 7). Tang testete bei dieser Reaktion außerdem Rhodium- und Kupferkatalysatoren und stellte fest, dass der Katalysator die Regioselektivität des Bindungsbruchs im Cyclopropanring (mit $\mathrm{R}^{1}=\mathrm{H}, \mathrm{R}^{2}=\mathrm{Ar}, \mathrm{R}^{3}$ $=\mathrm{H}$ ) beeinflusst. Die Ursachen dieser unterschiedlichen Regioselektivität sind nicht vollkommen ver- 


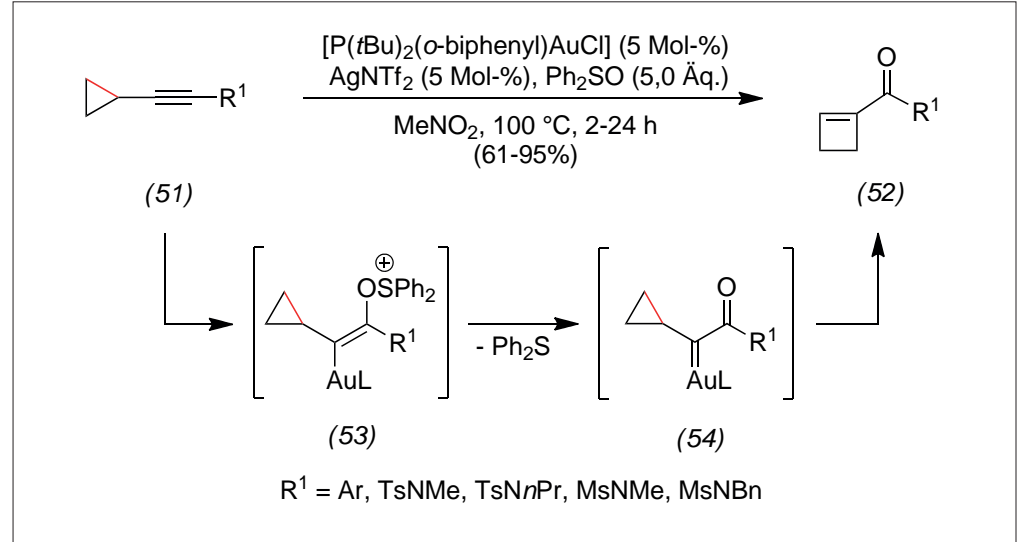

Abb. 8. Oxidative Ringerweiterung von Alkinylcyclopropanen nach Liu et al.

standen, müssen aber in den unterschiedlichen elektronischen und sterischen Effekten der verschiedenen Cyclopropyl-Metall-Carbene zu finden sein. ${ }^{12)}$

Eine oxidative Ringerweiterung führten Liu et al. an Alkinylcyclopropanen (51) mit einem Goldkatalysator durch. Als Sauerstoffquelle diente Diphenylsulfoxid, das im ersten Schritt des angenommenen Mechanismus zusammen mit dem Goldkatalysator an die Dreifachbindung addiert und somit das Intermediat (53) bildet. Nach dem Abspalten von Diphenylsulfid und der Generierung des Cyclopropyl-MetallCarben-Intermediats (54) erfolgt unter Abspaltung des Goldkatalysators die Ringerweiterung, die als Produkt Cyclobutenylketone (52) liefert. Um die Ausbeuten zu verbessern, war die Zugabe von $\mathrm{AgNTf}_{2}$ erforderlich. Es konnte zwar selbst die Reaktion nicht katalysieren, diente aber zum Austausch des Gegenions (Abbildung 8). ${ }^{13)}$

\section{Zusammenfassung}

Kleine Ringe als reaktive $C_{3}$ - oder $\mathrm{C}_{4}$-Bausteine finden immer wieder neue Anwendungen und bieten unzählige Möglichkeiten, schnell und effizient größere Zyklen aufzubauen. Somit zeigen sie ein großes Potenzial sowohl in der Naturstoffsynthese als auch in der pharmazeutischen Chemie. Da die Substrate in der Regel einfach herstellbar oder gar kommerziell erhältlich sind, sind Ringerweiterungsreaktionen nicht nur eine akademische Spielwiese.
Man darf in Zukunft gleichermaßen und auf die Anwendung schon bekannter Reaktionen in der Synthese komplexer organischer Moleküle gespannt sein.

Blickpunkt Synthese will zur Beschäftigung mit neuen synthetischen Verfahren oder eleganten und wichtigen Synthesen aus der Literatur anregen. Die Rubrik betreut in diesem Jahr Daniel B. Werz. Er ist Emmy-NoetherNachwuchsgruppenleiter an der Universität Göttingen. Bei diesem Beitrag unterstützte inn Johannes Kaschel. Er ist Doktorand in der Arbeitsgruppe von Daniel B. Werz.

\section{dwerz@gwdg.de}

\section{- Organische Synthese: Trends und Entwicklungen \\ Burkhard König organisiert die Vortragsreihe „Advances in Or- ganic Synthesis" auf dem GDCh- Wissenschafts-

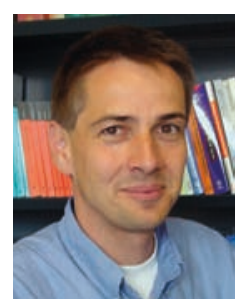 forum in Bremen - und präsentiert damit Vorträge nicht nur für „Voll- blutorganiker".}

Nachrichten aus der Chemie: Herr König, was erwartet den Besucher in der Session?

Burkhard König: Die eintägige Session gibt einen spannenden Überblick zu neuen Trends und Entwicklungen und spannt den Bogen von der Metallorganik und Katalyse über Naturstoffe bis zur supramolekularen Chemie. Vollblutorgaauf neue Ringöffnungsreaktionen
Literatur

1) A. D. Walsh, Nature $1947,159,712-713$.

2) a) H.-U. Reissig, R. Zimmer, Chem. Rev. 2003, 103, 1151-1196; b) C. A. Carson, M. A. Kerr, Chem. Soc. Rev. 2009, 38, 3051-3060.

3) A. T. Parsons, J. S. Johnson, J. Am. Chem. Soc. 2009, 131, 3122-3123.

4) A. T. Parsons, J. S. Johnson, J. Am. Chem. Soc. 2009, 131, 14202-14203.

5) H. K. Grover, T. P. Lebold, M. A. Kerr, Org. Lett. 2011, 13, 220-223.

6) M. M. A. R. Moustafa, B. L. Pagenkopf, Org. Lett. 2010, 12, 4732-4735.

7) A. C. Stevens, C. Palmer, B. L. Pagenkopf, Org. Lett. 2011, 13, 1528-1531.

8) M. Gulias, J. Durán, F. López, L. Castedo, J. L. Mascareñas, J. Am. Chem. Soc. 2007, 129, 11026-11027.

9) L. Saya, G. Bhargava, M. A. Navarro, M. Gulias, F. López, I. Fernández, L. Castedo, J. L. Mascareñas, Angew. Chem. 2010, 122, 10082-10086.

10) T. Seiser, N. Cramer, Angew. Chem. 2008 , 120, 9435-9438.

11) T. Seiser, O. A. Roth, N. Cramer, Angew. Chem. 2009, 121, 6438-6441.

12) H. Xu, W. Zhang, D. Shu, J. B. Werness, W. Tang, Angew. Chem. 2008, 120, 9065-9068.

13) C.-W. Li, K. Pati, G.-Y. Lin, S. M. A. Sohel, H.-H. Hung, R.-S. Liu, Angew. Chem. 2010, 122, 10087-10090. 